Perspectiva Geográfica

ISSN 0123-3769 (Impreso) - 2500-8684 (En línea)

Vol. 21 N. ${ }^{\circ} 2$ de 2016

Julio - Diciembre

pp. 277-298

\title{
Gestión integral de la industria cauchera en el municipio de San José del Guaviare, Colombia
}

\author{
Integral Management for the Rubber Industry in the \\ Municipality of San José del Guaviare, Colombia
}

\section{Grace Andrea Montoya Rojas ${ }^{1}$ \\ Adriana Posada ${ }^{2}$ Johana Martín ${ }^{3}$ Laura Garnica ${ }^{4}$ Pilar Peña ${ }^{5}$ Ana Ramírez ${ }^{6}$}

Para citar este artículo utilice el nombre completo así:

Montoya-Rojas, G., et. al. (2016). Gestión integral de la industria cauchera en el municipio de San José del Guaviare, Colombia. Perspectiva Geográfica, 21(2), 277-298. doi: $10.19053 / 01233769.5854$

\section{Resumen}

En el artículo se plasman los logros de un ejercicio investigativo mediante una innovación espacial aplicada de manera integral a la industria del Caucho. De

\footnotetext{
Agróloga, Ph.D. Docente Investigadora U.D.C.A Ingeniera Geográfica y Ambiental. graceandreas@gmail.com

2 Economista Agrícola, MSc., Docente Investigadora U.D.C.A. Ingeniera Geográfica y Ambiental. adriaposada@yahoo.es

3 Ingeniero Geógrafo y Ambiental, U.D.C.A, Bogotá, Colombia. johanandrea2@gmail.com

4 Ingeniero Geógrafo y Ambiental, U.D.C.A, Bogotá, Colombia. catii1206@gmail.com

5 Ingeniero Geógrafo y Ambiental, U.D.C.A, Bogotá, Colombia. pilarp0406@gmail.com

6 Ingeniero Geógrafo y Ambiental, U.D.C.A, Bogotá, Colombia. annieramirez.92@hotmail.com
} 
esta forma, se promueve una planificación holística en el municipio de San José del Guaviare, Colombia, donde hay actividades de colonización en la región selvática y, además, se encuentran comunidades indígenas y variedad de resguardos. La gestión integral de la agroindustria del Caucho, uno de los medios de susbsistencia de los habitantes, es prácticamente ausente. El proyecto cuenta con un diagnostico general de la zona, mediante el cual se obtienen modelaciones SIG con el fin de establecer las áreas potenciales para la producción de neumáticos en la agroindustria de Caucho. Se ilustra desde la gestión integral sectorial, las áreas capaces de soportar el proyecto de manera productiva y lograr al mismo tiempo, un desarrollo armónico entre el territorio municipal y el proyecto.

Palabras clave: análisis espacial, caucho, escenarios, gestión ambiental, modelos, planeación, Sistema de Información Geográfica, sostenibilidad.

\section{Abstract}

The achievements of this research exercise are reflected by a spatial innovation, applied comprehensively to the rubber industry. In such a way, holistic planning is promoted in the municipality of San José del Guaviare, Colombia, where there are settlement activities in the jungle region and also native communities and variety of safeguards. The comprehensive management of the rubber agribusiness, one of the means of subsistence for the inhabitants, is practically absent. The project performed an overall diagnosis of the area, through GIS modeling in order to establish potential areas for the production of rubber tire agribusiness. As illustrated from the integrated management sector, areas are shown which are capable of supporting the project productively and at the same time achieving a harmonious development between the municipal territory and the project.

Keywords: spatial analysis, rubber, scenarios, environmental management, modeling, planning, Geographic Information Systems, sustainability. 


\section{Introducción}

Este artículo es el resultado de un ejercicio académico que zonifica áreas para orientar el desarrollo de una industria cauchera en el municipio de San José del Guaviare. Según Martínez (2005), el caucho natural tuvo un uso masivo tiempo atrás, basado en la fabricación de jarras y tubos, esto paulatinamente fue ganando reconocimiento hasta la eventual fabricación de ropa. En la actualidad, su producción es importante en Colombia ya que tiene un gran auge y comercialización para el país.

La extracción del caucho consiste principalmente en sustraer el látex en plantas productoras a través de fases como la extracción, la transformación a neumático y a medio de pavimentación. Finalmente se hace referencia al medio por el cual se pretende establecer un equilibrio entre los recursos naturales renovables y el medio ambiente. El ciclo productivo del caucho permite determinar los riesgos, normas, programas, planes, proyectos y certificaciones que soportan el desarrollo del proyecto, además de establecer con mayor detalle la gestión ambiental que debe ejercerse sobre cada dimensión territorial y cada componente ambiental, y de esta manera lograr la ejecución y el desarrollo óptimo del proyecto.

San José del Guaviare es un municipio que cuenta con características naturales propicias para el desarrollo cauchero, y tiene varios proyectos de extracción natural de Caucho. Sin embargo, no cuenta con una debida gestión ambiental de los recursos impactados ni infraestructura que brinde la comercialización adecuada del producto, esto se debe a la falta de desarrollo en el municipio y la escases de información y análisis antes de la ejecución del proyecto. (Alcaldía San José del Guaviare, 2011).

Como parte del trabajo realizado, se generó un análisis de las dinámicas territoriales comprendidas en el municipio, de los impactos y riesgos que este puede ocasionar, y un diagnóstico general de la zona logrado mediante la integración de los Sistemas de Información Geográfica (SIG). El artículo tiene como objetivo mostrar una modelación para la gestión integral de la industria cauchera en el municipio de San José del Guaviare, Colombia. 
Partiendo de la premisa de que el ordenamiento territorial es un proceso que utiliza diversas estrategias e instrumentos para lograr una relación armónica población-territorio (Posada y Berrocal, 2015), al aplicarlo a un caso concreto es necesario considerar diversos criterios u objetivos en ámbitos espaciales de diferente índole. Esto exige a su vez, aplicar instrumentos como los SIG y la evaluación multicriterio, de manera que permita extrapolar escenarios capaces de involucrar diversas variables, lo cual permitirá enriquecer los resultados pretendidos. (Mena et al., 2006). En éste mismo orden de ideas, Posada et al. (2016) hacen énfasis en que se puede utilizar alguna representación esquemática de los elementos de un sistema, y de las relaciones que existen entre ellos a la hora de desarrollar escenarios y modelos, por lo cual, la gestión integral exige detallar los procesos de integración, de relaciones e interacciones externas e internas del sistema territorial.

\section{Planteamiento metodológico}

La modelación como propuesta de la gestión integral del Caucho, se realizó en las siguientes seis etapas:
Etapa 1. Construcción de la Visión Territorial: inicialmente, para la ejecución del proyecto se realiza la revisión de información secundaria para la interpretación y análisis de resultados relacionados a la extracción de caucho natural en el municipio de San José del Guaviare. Se hace una breve descripción de los componentes ambientales (atmosférico, hidrosférico, gesoférico y bisférico), el componente antroposférico se analiza desde las dimensiones $^{7}$ de la sostenibilidad las cuales incluyen la dimensión ambiental, económica, social, institucional y gubernamental. Esta visión territorial, hace referencia a la línea base que constituye el insumo de análisis del modelo propuesto.

Etapa 2. Entendimiento del proyecto articulado a la Oferta del Territorio - OT: con base en la visión territorial construida, se propone un análisis organizado por matri$\operatorname{ces}^{8}$ del uso y aprovechamiento de

7 Trascendiendo a la trilogía de la sostenibilidad (ambiente, sociedad y economía), se requiere incorporar los motores que articulan dicha trilogía, que, con base en la experiencia sectorial y la dinámica de cada territorio, materializa y sale al paso con el apoyo institucional y un marco legal y político sincronizado. (Montoya, 2016).

8 El análisis multicriterio representado en matrices funciona muy bien cuando se requiere organizar la información por unidades de análisis, tal como propone Montoya (2016) en el modelo de Gestión Integrada 
los recursos naturales demandados por la industria del Caucho en las distintas fases del ciclo productivo del Caucho.

Etapa 3. Modelación para determinar Áreas Potenciales Prioritarias - APP: se contemplaron las entradas como las características ambientales presentes en cada una de los factores que integran los cuatro componentes ambientales, destacando como prioridades altas aquellas zonas que resultan óptimas para el desarrollo e implementación de la actividad de extracción de Caucho, permitiendo de esta manera, determinar la zona propicia para su ubicación.

Bajo el criterio de las condiciones brindadas por el territorio para la actividad, se clasificó en tres rangos diferentes: alto cuando las características del medio eran idóneas para la ejecución de la actividad; medio cuando dichas características variaban en algunos componentes pero a pesar de esto sobresalían condiciones de algunos que permitían el desarrollo de la actividad; y bajo cuando los recursos naturales presentes en el

del Territorio, para el manejo de la información por componentes ambientales, por dimensiones, por fase de proyecto en el ciclo productivo del mismo. territorio no eran los ideales para la realización de la extracción del Caucho. Dichos rangos fueron categorizados en tres colores: Alto en rojo, medio en amarillo y bajo en verde.

Para establecer la categorización de APP para cada componente, se superpuso cada uno de los factores ya categorizados, donde la ponderación hecha entre cada uno de los rangos siendo bajo equivalente a 1 , medio $2 \mathrm{y}$ alto 3 tomaba relevancia, el resultado de dicha ponderación determinaba el rango para el componente. Dichos rangos fueron establecidos de la siguiente manera: bajo de 1 a 1.5; medio de 1.6 a 2.5 y alto de 2.6 a 3. Finalmente, para obtener el mapa de APP se superponen los cuatro componentes ambientales ya categorizados siguiendo la metodología mencionada anteriormente para la clasificación de las APP por municipio de la Dimensión Ambiental, mediante los SIG (Alberti y Wadell, 2000). ${ }^{9}$

9 Alberti y Wadell (2000) mencionan que los modelos de desarrollo urbanos o rurales, sirven como instrumentos de monitoreo y control para las autoridades territoriales. Aspecto que como futura línea de investigación se podría plantear teniendo como base este trabajo de caucho, para establecer una gestión adecuada de la oferta territorial a corto, mediano y largo plazo. 
Etapa 4. Modelación para la Planeación Espacial Estratégica $P E E$ : para la consolidación del este mapa, se tienen en cuenta los elementos funcionales ${ }^{10}$ (Sandström et al., 2016) del municipio de San José del Guaviare, destacándose además los diferentes comportamientos de la estructura de la planeación que van desde lo nodal, lineal, hasta lo mixto en aquellas zonas de desarrollo urbano y económico, soportadas por vías de comunicación tanto terrestre como fluvial.

Etapa 5. Referente Normativo Estratégico del proyecto - RNE: para la ejecución del proyecto de extracción del caucho en el municipio de San José del Guaviare, en esta etapa se elabora un normograma con una serie de normas nacionales e internacionales, políticas, leyes, decretos y resoluciones que soportan el desarrollo del proyecto en cada fase del ciclo productivo del caucho.

Etapa 6. Zonificación de la Gestión Integral: donde se identifican una

10 Los elementos funcionales son aquellos que permiten entender las necesidades humanas que inducen a la transformación del paisaje urbano-rural. Estos siete elementos son dinámicos en el tiempo y permiten establecer tendencias de desarrollo y movilidad, tales son: vivienda, formas de trabajo, servicios, transporte, identidad, desarrollo estructural, infraestructura. (Integrate Urban Planing Sweeden, 2007). serie de planes, programas y proyectos para cada dimensión establecida. La dimensión ambiental corresponde a lo realizado con las APP, la dimensión institucional se analiza con la propuesta de la PEE y la dimensión gubernamental corresponde al RNE. Los planes, programas y proyectos que se establecen en la tabla hacen referencia a aquellos que ya se están ejecutando en el municipio de San José del Guaviare, y algunos de los que se pretenden desarrollar a un futuro. En esta etapa se presenta el resultado de la factibilidad del proyecto con base en el modelo SIG propuesto, para espacializar la factibilidad de un proyecto con base en los criterios de la gestión integral del territorio.

\section{Resultados}

La modelación territorial para la gestión integral de la industria cauchera en el municipio de San José del Guaviare se logró con los siguientes seis resultados:

\subsection{Construcción de la Visión Te- rritorial}

Desde la construcción de la sostenibilidad sectorial y un contexto globalizado, se propone agrupar en 
los aspectos dinamizadores del territorio como son la dimensión ambiental, social, económica, institucional y gubernamental, tal como se precisa a continuación:

Dimensión ambiental: sobre el componente atmosférico, geosférico, hidrosférico, biosférico.

Dimensión social: temas relacionados con la Geografía Histórica, el Plan Básico de Ordenamiento Territorial, el proceso de colonización, la dinámica poblacional y otros datos del Departamento Administrativo Nacional de Estadística (2005).

Dimensión económica: ${ }^{11}$ economía tradicional, economía de autoconsumo e intercambio de productos en la mayoría del territorio debido a la presencia de grupos indígenas (Diócesis del Guaviare, s. f.).

Dimensión institucional: pese a la infraestructura poco adecuada para la ejecución de grandes proyectos, establecer medios de comunicación que elevan el costo y por ende el acceso de comercialización de productos en la zona ya que se caracteriza por comunicar gran parte del país. Algunas de las empresas y colaboradores dentro del municipio para el desarrollo del proyecto se encuentran en la Figura 1 , donde se consolida la información consultada en el instituto Colombiano Agropecuario, el Instituto Colombiano de Investigaciones Científicas, el Instituto de Hidrología, Meteorología y Estudios Ambientales de Colombia (2014), el Ministerio de Tecnologías de la Información y las Comunicaciones (2013), y el Ministerio de Interior (2005).

Dimensión gubernamental: el municipio presenta una serie de políticas, normas y buenas prácticas establecidas para el desarrollo del proyecto. Para esta visión gubernamental o de gobernanza en un tipo de proyectos industriales es muy importante considerar no solo el diálogo de saberes con los intereses en juego, sino el cumplimiento de las normas ISO 26000 que toma el ejercicio con los grupos de interés del entorno de la industria.

11 Según Fernández (2013), la dimensión económica requiere un análisis integral para establecer una propuesta integral y sostenible en el territorio. 


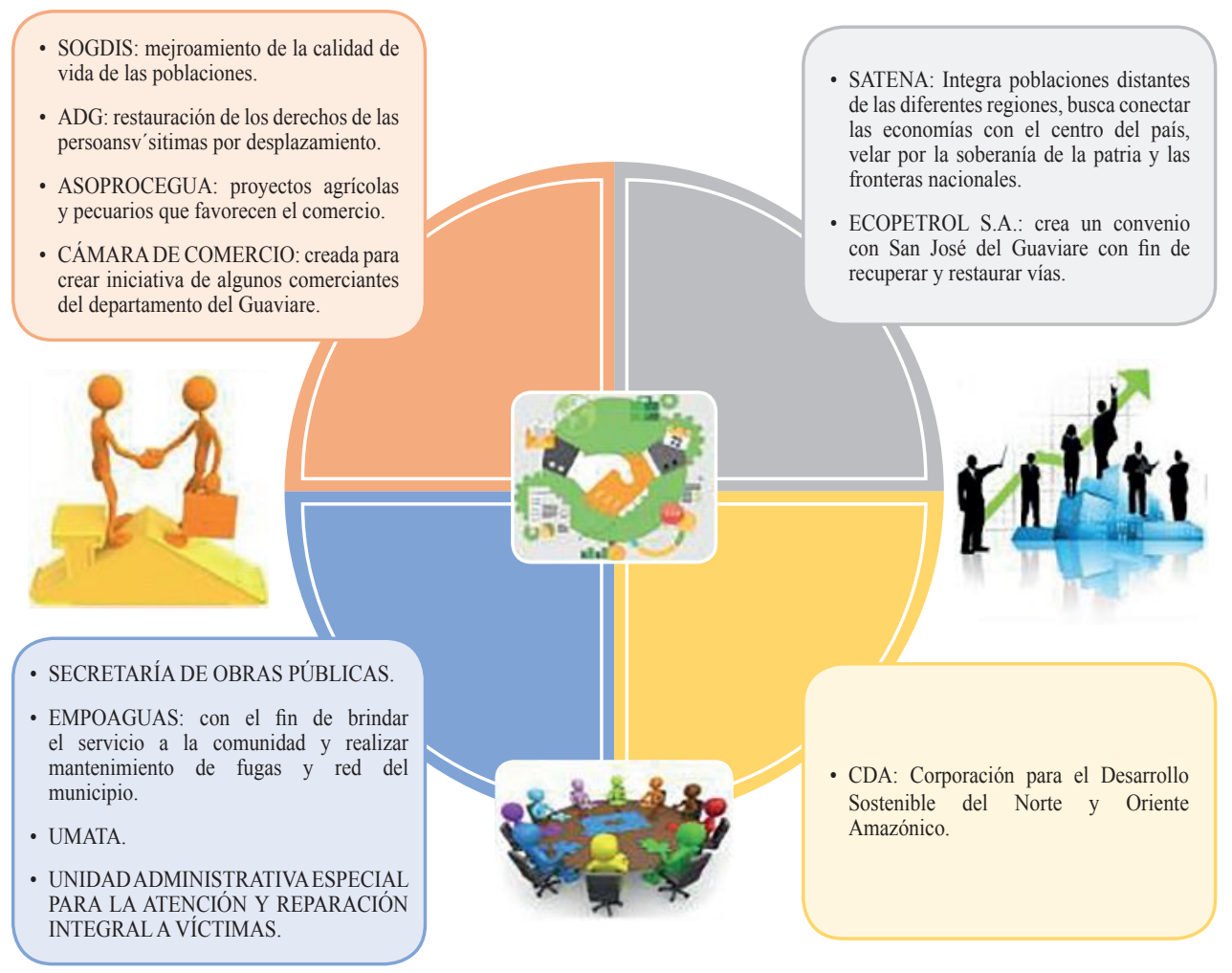

Figura 1. Algunas empresas y colaboradores del municipio para el desarrollo del proyecto

Fuente: Elaboración propia.

3.2 Entendimiento del proyecto de su ciclo productivo. El caucho articulado a la Oferta del Terri- es materia prima fundamental para torio

Con base en la visión territorial construida, se describe el ciclo productivo del proyecto por fases, y se propone un análisis organizado por matrices del uso y aprovechamiento de los recursos naturales demandados por la industria del caucho en las distintas fases productos de gran escala mundial como los neumáticos, y San José del Guaviare cuenta con zonas potenciales aptas para estos cultivos debido a las características edafológicas y climáticas. Por esta razón se busca implementar este cultivo por ser un elemento importante en la reforestación y cuidado ambiental, implantándolo en planes de de- 
sarrollo municipales, con el fin de multiplicar la siembra generando una reforestación de suelos previamente usados en la ganadería y así consolidar a San José del Guaviare como uno de los municipios pioneros en la producción de caucho, generando inversión ya sea nacional o internacional (Ver Figura 2).

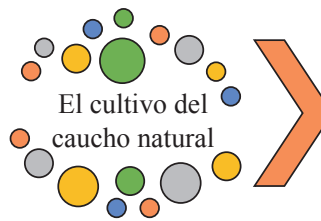

$\begin{array}{ll}\begin{array}{ll}\text { - Importante subsector de } \\ \text { la economía nacional. }\end{array} & \begin{array}{l}\text { Producción continua } \\ \text { durante } 25 \text { o } 30 \text { años. }\end{array} \\ \begin{array}{ll}\text { - Clave para el comercio } \\ \text { internacional. }\end{array} & \begin{array}{l}\text { Materia prima con una } \\ \text { demanda creciente e } \\ \text { insatisfecha. }\end{array}\end{array}$
El cultivo del caucho posee particularidades que son únicos

- Importante elemento de reforestación y cuidado ambiental.

- El gobierno municipal lo incluyó como tema prioritario en los planes de desarrollo municipales.
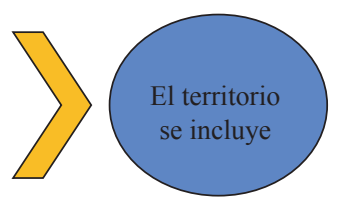

$$
\begin{aligned}
& \text { - Oportunidad para } \\
& \text { multiplicar la siembra } \\
& \text { y generar una } \\
& \text { reforestación. } \\
& \text { - San José de Guaviare } \\
& \text { se constituya como } \\
& \text { uno de los cultivadores } \\
& \text { importantes a nivel } \\
& \text { nacional. }
\end{aligned}
$$

Figura 2. Esquema sobre la articulación del proyecto y la articulación con el Territorio Fuente: Elaboración propia.

Para proponer una gestión integral en el territorio, en el ciclo del caucho (desde su punto de partida hasta la producción final de diversos productos) el propósito del proyecto de gestión se centró en la extracción del caucho natural, pasando por la trasformación de la materia prima hasta la producción de neumáticos y finalmente la reutilización de estas en pavimento.

El modelo de "Producción en Ciclo Cerrado" busca no solo racionalizar el uso de los materiales y recursos, sino también orientar un primordial enfoque en el diseño de productos y servicios, compensando de una u otra forma los impactos que se ocasionan con la aplicación de determinado proyecto (ECPA, s.f.). Por esta razón, para la extracción de caucho en San José del Guaviare se implantan diferentes fases de extracción, transformación, re-utilización y compensación de las actividades realizadas, como se evidencia en la Figura 3 (Altervista, 2009). 


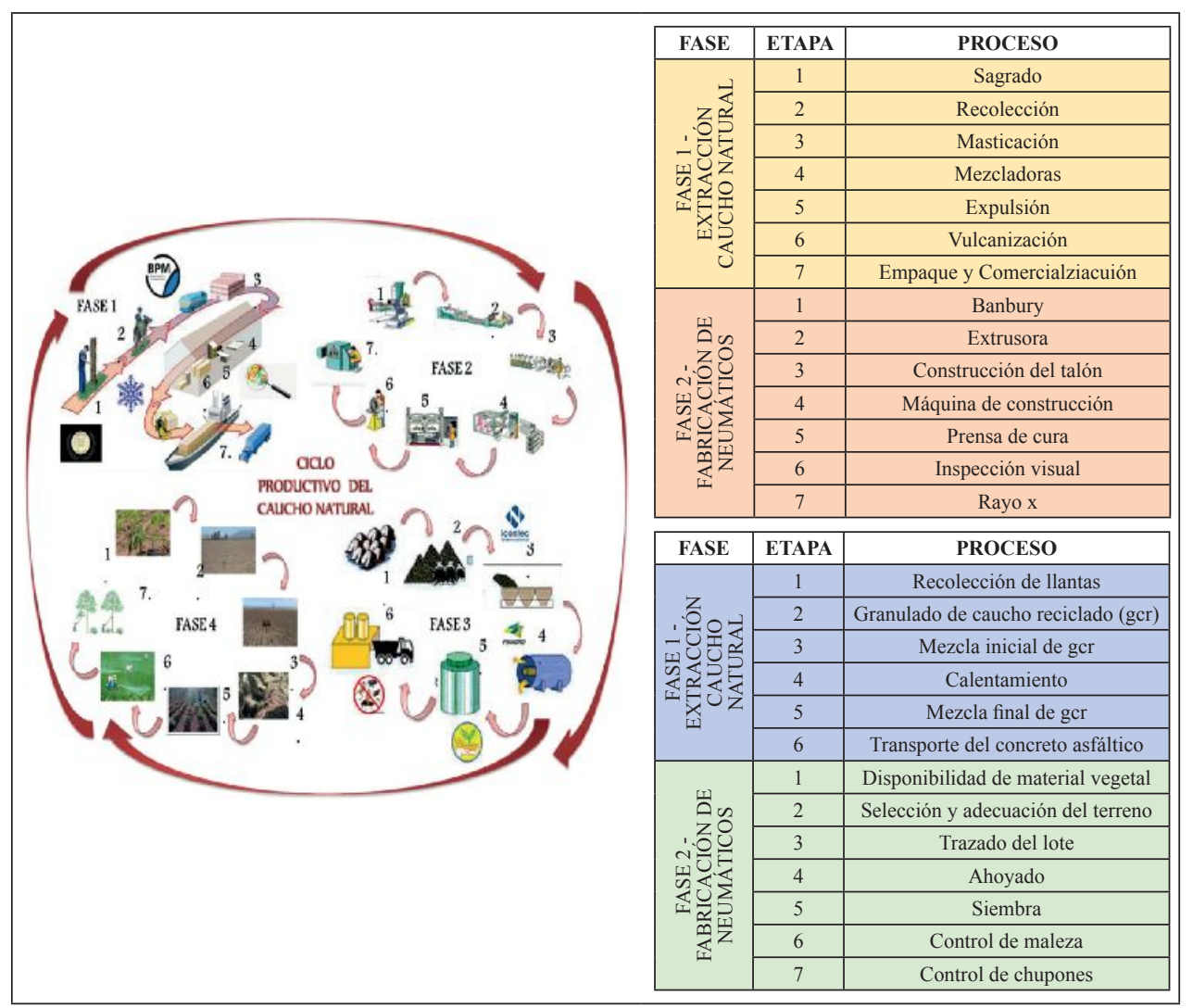

Figura 3. Esquema ilustrativo del ciclo productivo y las fases a las que se le hizo un análisis de uso y aprovechamiento de los recursos naturales

Fuente: Elaboración propia.

3.2.1 Análisis de uso y aprove- territorio en la dimensión ambienchamiento de los recursos natu- tal y sus componentes. rales del Proyecto

Teniendo como base cada uno de fases de la producción de caucho los procesos del ciclo productivo generan un aprovechamiento puesse hizo un análisis de uso y aprove- to que se busca reciclar y reutilizar chamiento a partir de la demanda los recursos, un ejemplo de estos de recursos que el proyecto requie- es el agua utilizada en el proceso re, además, yace la sospecha de de sangrado, recolección y mastique tanta presión se genera en el cación, la cual es reutilizada en el 
lavado de vasijas y recipientes generando un costo bajo y por ende un proceso optimo. Sin embargo, el proceso del caucho en general produce un gran aprovechamiento de recursos del componente geoesférico, hidrosférico y biosférico, esto se debe a que al no ser zonas establecidas por la autoridad ambiental no cuentan con un proceso técnico, generando una alta alteración del suelo y del aire, y así, afectando las propiedades físicas y químicas del suelo. Todo esto ocasiona consecuencias a largo plazo en la comunidad (como infertilidad y enfermedades respiratorias para los trabajadores) y de esta manera se genera un alto costo de inversión a la hora de implementar este cultivo en esta zona.

\subsection{Modelación para determinar} Áreas Potenciales Prioritarias APP

Para la modelación de las APP se establecen criterios de evaluación en un rango de alto, medio y bajo, tal como se había mencionado para la realización de la extracción de caucho. Estos rangos fueron categorizados en tres colores: Alto en rojo, medio en amarillo y bajo en verde. La Figura 4 ilustra los resultados de la modelación por componente ambiental y la Figura 5 muestra las APP como resultado de aplicar el modelo de Sistemas de Información Geográfica en la dimensión ambiental.

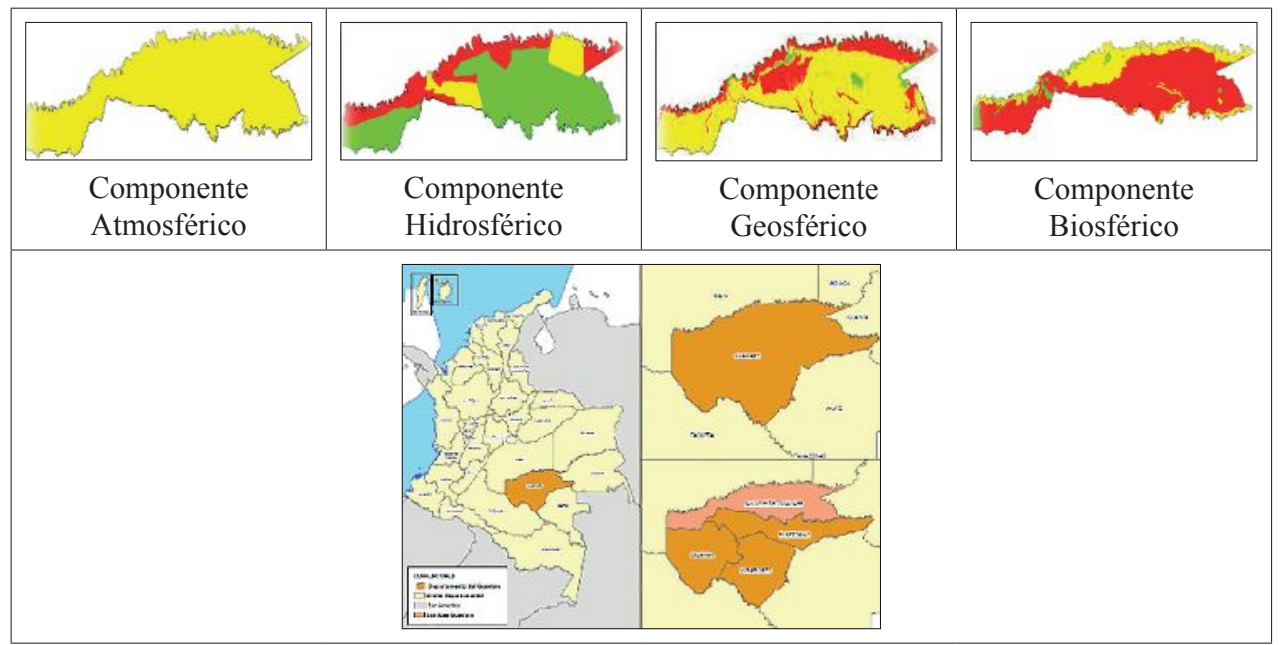

Figura 4. Resultado de las Áreas Potenciales Prioritarias por cada uno de los Componentes Ambientales para el proyecto

Fuente: Elaboración propia. 


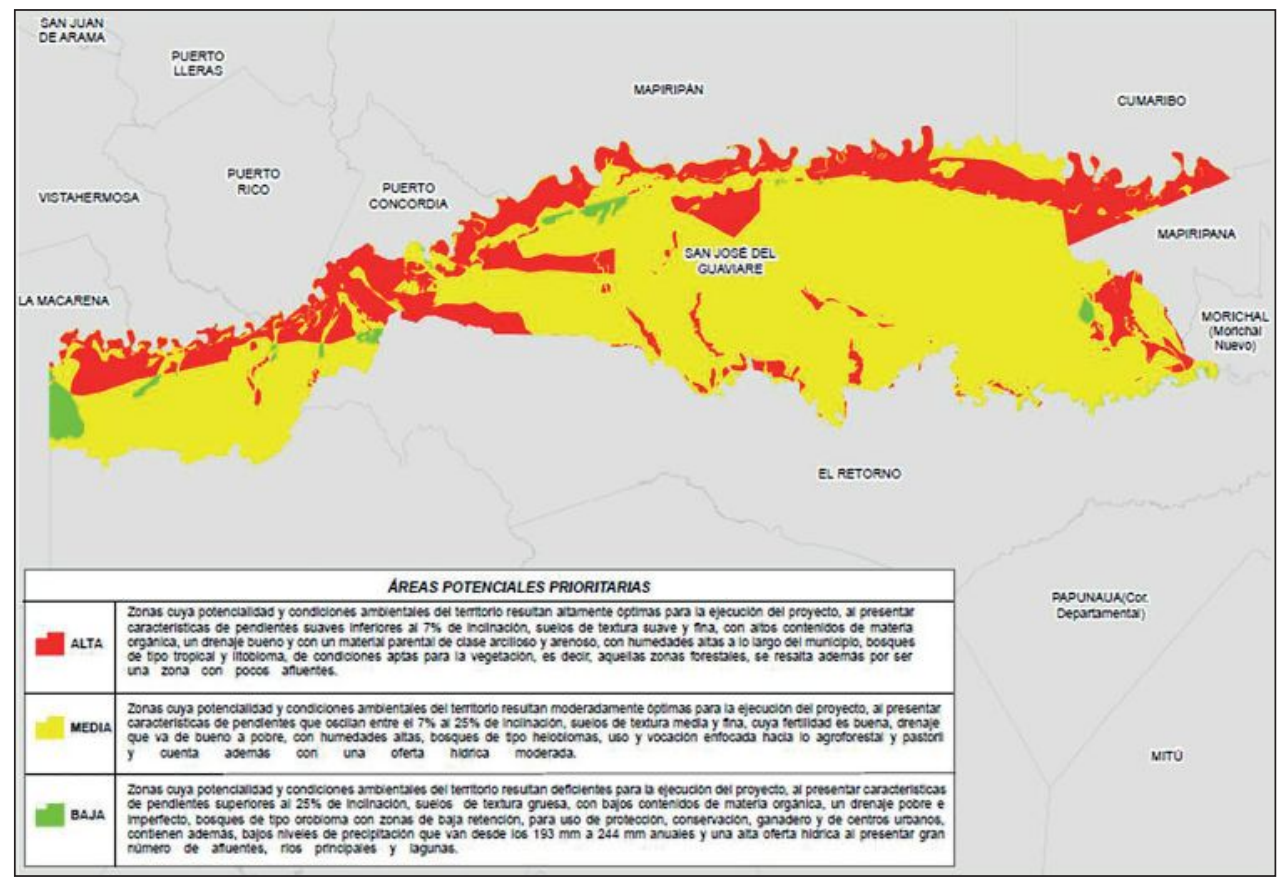

Figura 5. Resultado de las Áreas Potenciales Prioritarias de la dimensión ambiental para la zona del proyecto

Fuente: Elaboración propia.

3.4 Modelación para la Planeación Espacial Estratégica - PEE Teniendo en cuenta los elementos funcionales del Municipio de San José del Guaviare, se destacan los diferentes comportamientos de la estructura de la planeación que van desde lo nodal, lineal, hasta lo mixto en aquellas zonas de desarrollo urbano y económico soportadas por vías de comunicación tanto terrestre como fluvial. Dichas características permitieron establecer la dinámica territorial existente en el municipio con el desarrollo y la ejecución del proyecto de Cauchos
Capijoma S. A. Los elementos funcionales son establecidos a partir de información secundaria recopilada y el ejercicio académico (ver Figura 6) correspondiente a PEE, ubicando cada uno de los elementos funcionales en el municipio.

\subsection{Referente Normativo Estra- tégico del Proyecto - RNE}

Para la ejecución del proyecto de extracción del caucho en el municipio de San José del Guaviare, se cuenta con una serie de normativas tanto nacionales como internacio- 
nales que requieren de su imple- mantienen su labor durante todas mentación desde antes del funcio- las fases contenidas en el ciclo pronamiento del proyecto y otras que ductivo.

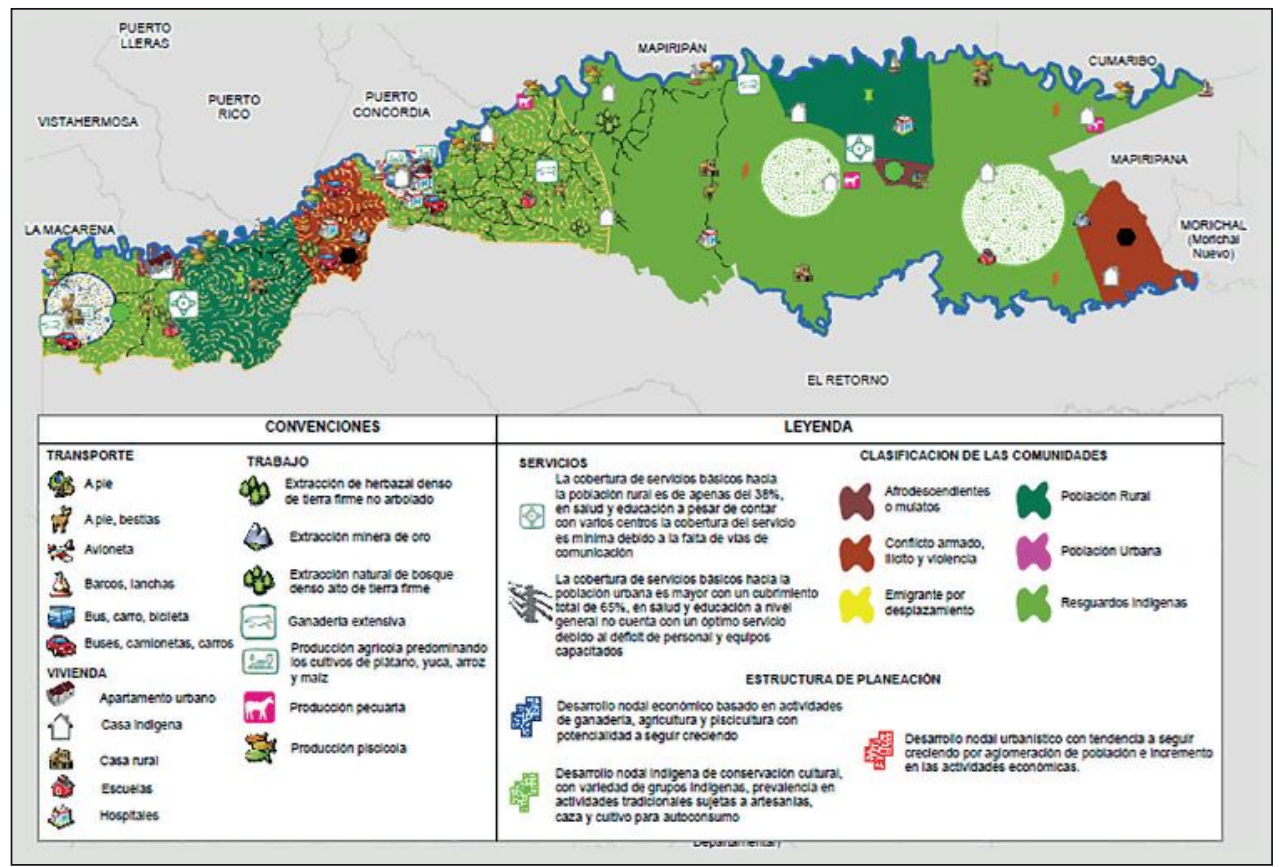

Figura 6. Resultado de las Planeación Espacial Estratégica que ilustra la dimensión social y económica para la zona del proyecto

Fuente: Elaboración propia.

3.6 Zonificación de la Gestión Integral - ZGI

Esta etapa corresponde al ejercicio de identificar una serie de planes, programas y proyectos para cada dimensión establecida. La dimensión ambiental corresponde a lo realizado con las APP, la dimensión institucional se analiza con la propuesta de la PEE y la dimensión gubernamental corresponde al RNE.
La Figura 8 es un esquema correspondiente al mapa de actores. Se determina a partir de la clasificación de tres actores principales en los cuales se conforma la participación de actores clave durante el desarrollo del proyecto. Como actores influyentes se determinan aquellos de gran importancia en la parte funcional del proyecto, hacen referencia a los directores del departamento empresarial, accionistas, socios comerciales y trabaja- 
dores quienes conforman Cauchos Capijoma S. A. Los actores distantes pero influyentes hacen referencia a aquellos que indirectamente proporcionan ayudas en programas, productos, capacitaciones $\mathrm{y}$ posicionamiento tales como agremiaciones caucheras, Invias, el Ministerio de Ambiente y Desarro1lo Sostenible, proveedores, entre otros, siendo a su vez influyentes por la generación de estas activida- des. Finalmente, los actores significantes e influyentes son aquellos que influyen en el mercado nacional del producto y a su vez establecen una significancia que mide la capacidad productiva del proyecto aumentando su comercio o disminuyendo el mismo, tales como la comunidad y los consumidores o clientes. El conjunto de estos actores consolida el óptimo desarrollo del proyecto.

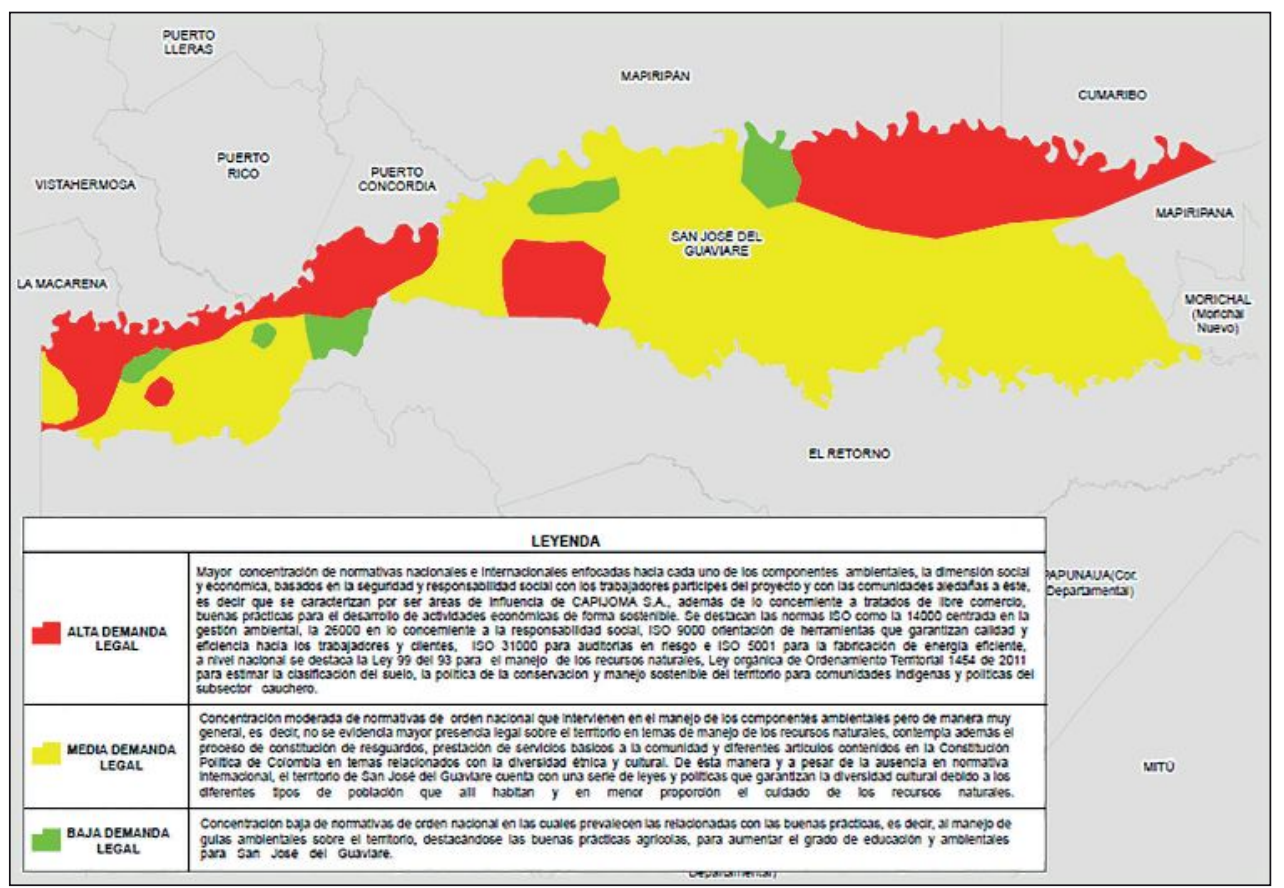

Figura 7. Resultado del ejercicio de espacializar la normativa en el municipio, con base en la estructuración del normograma, de esta manera se representa la dimensión gubernamental de la sostenibilidad para la zona del proyecto

Fuente: Elaboración propia.

Cabe resaltar que las agremiaciones, asociaciones, consumistas y comunidad no representan un actor específico ya que son actores que pueden variar con el desarrollo del proyecto y no se sabe con exacti- 
tud el nombre de la persona o autor que colabora como soporte institucional en el proyecto. La Figura 9 espacializa la gestión del territorio con base en los planes, programas y proyectos en el municipio.

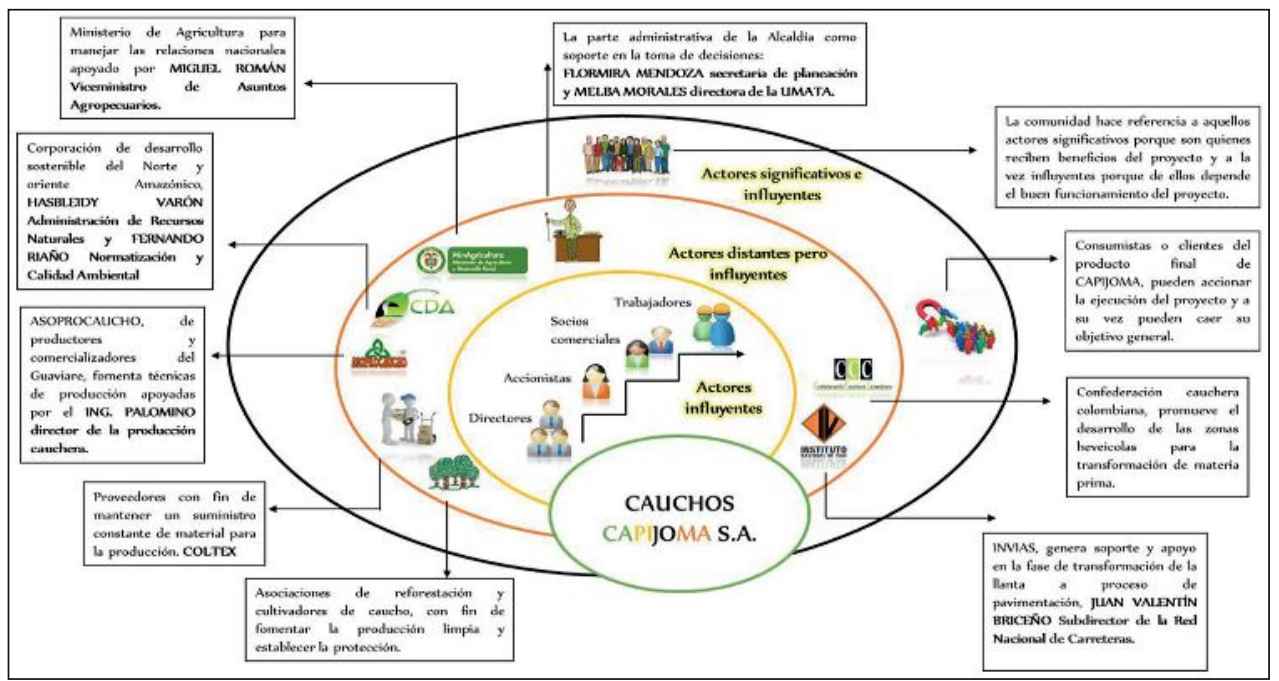

Figura 8. Mapa de actores analizado en la gestión integral del proyecto en el municipio Fuente: Elaboración propia.

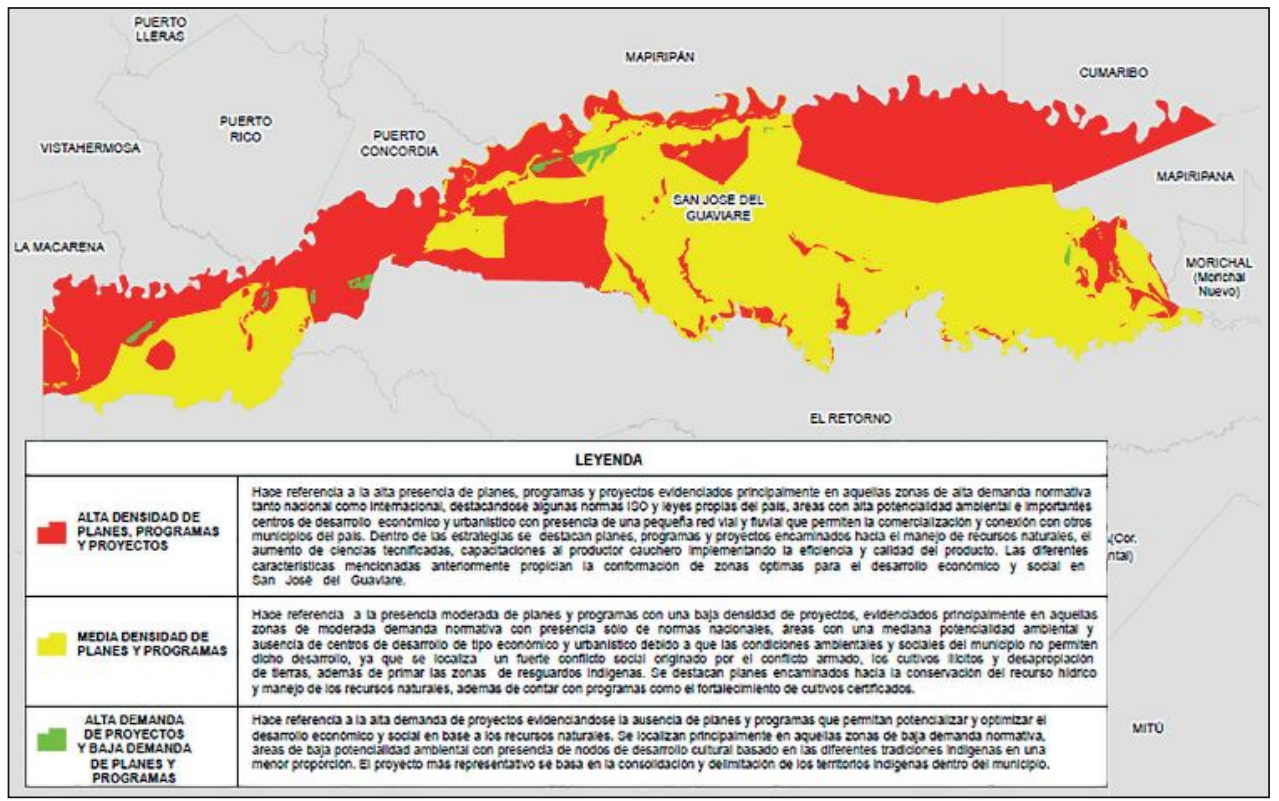

Figura 9. Mapa de zonificación de la gestión integral territorial

Fuente: Elaboración propia. 


\subsubsection{Zonificación de factibilidad del proyecto}

Después de realizar un estudio sistemático de la oferta del territorio por componentes ambientales y las dimensiones de la sostenibilidad, y tras realizar los respectivos cruces del modelo SIG propuesto para la gestión integral del territorio para el proyecto de Caucho en el municipio de San José del Guaviare, se realiza una zonificación de factibilidad. La factibilidad permite establecer la viabilidad final del proyecto a partir del cruce y análisis de los mapas y matrices elaborados anteriormente, partiendo de la inversión a realizar en cada una las zonas establecidas en el municipio bajo los parámetros de recursos naturales, infraestructura vial y urbana y la población, con el fin de establecer y determinar la ubicación más estratégica para cada una de las diferentes fases de ejecución del ciclo productivo.

El mapa de factibilidad evidencia la localización de cada una de estas, distribuyéndose de la siguiente manera:

Fase 1: extracción de caucho natural. Se ubica al suroccidente y oriente del municipio, la cual contempla manejar la norma ISO 22000, el certificado de buenas prácticas para la fabricación, la aplicación de medidas para reducir la temporada invernal y normas de carácter fitosanitario y de recursos biológicos para la comercialización, todo lo anterior contemplado en la certificación del ciclo productivo.

Fase 2: fabricación de neumáticos. Se ubica al sur del casco urbano, en ésta se contempla los certificados de compensación forestal regidos por el ICONTEC y los incentivos forestales que ofrece el Ministerio de Ambiente y Desarrollo Sostenible (2013; Goodyear, s. f.).

Fase 3: transformación de las llantas en pavimento. Se ubica al occidente del municipio, contempla los certificados de compensación forestal regidos por el ICONTEC, certificación de buenas prácticas de Manejo de suelos, certificado de incentivo forestal y servicio de gestión de plagas.

Fase 4: fabricación. Se ubica al suroccidente y centro sur del municipio, contempla ASTM 
Evaluación estándar de la producción de neumáticos, Normas ISO para la práctica y aprove- chamiento de llantas y neumáticos usados (ver Figura 10).

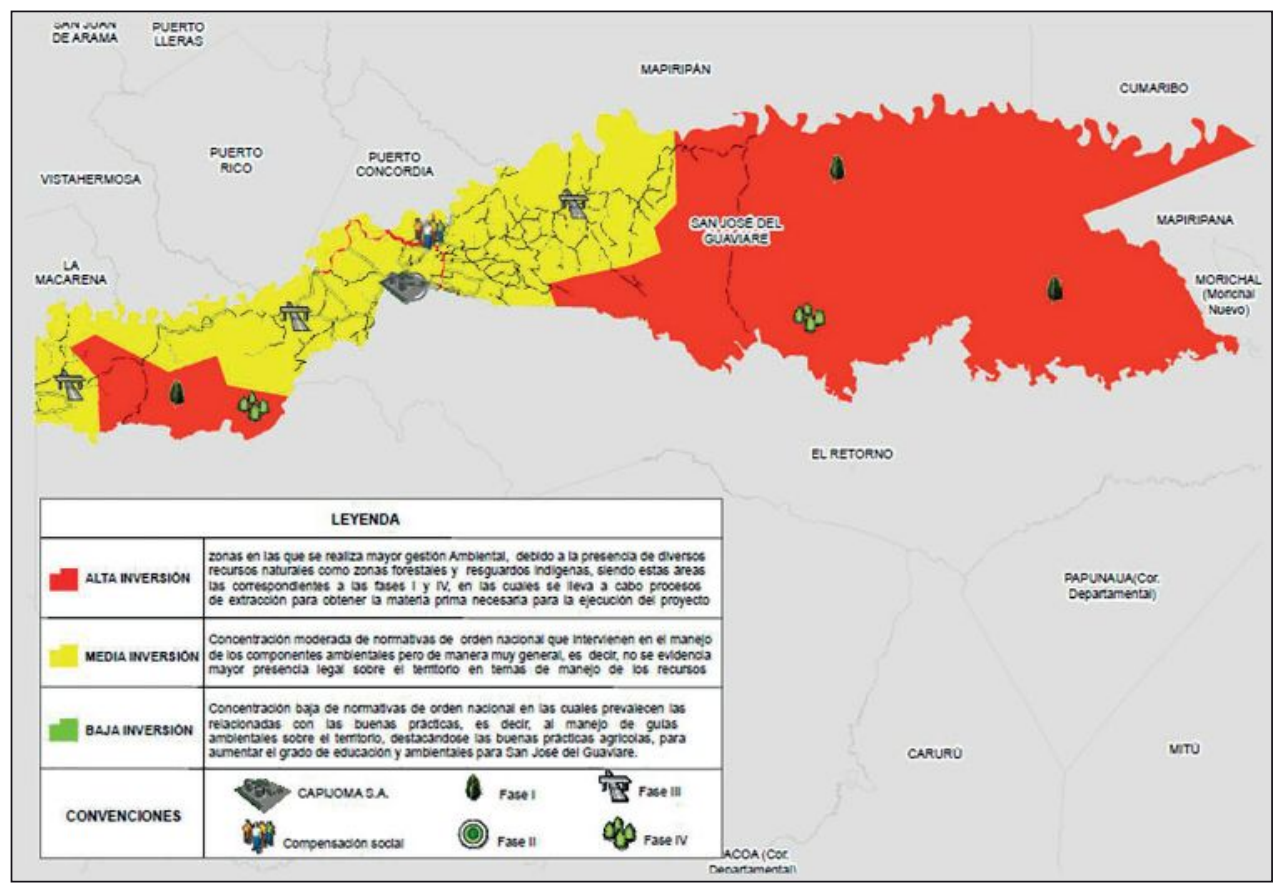

Figura 10. Mapa de zonificación de factibilidad del proyecto, donde se sugiere una ubicación territorial del desarrollo de las fases del ciclo productivo del Caucho Fuente: Elaboración propia.

\section{Discusión y conclusiones}

La modelación de la gestión integral de la industria cauchera en el municipio de San José del Guaviare, permitió establecer la factibilidad de esta sobre la zona sin causar daño ambiental. Debido al análisis exhaustivo no solo de los datos obtenidos a partir de la herramienta
SIG sino además de la información secundaria recopilada, así como de la sobre-posición de diferentes capas de información de índole ambiental, social, económico, cultural, entre otras, se logró establecer las diversas zonas óptimas para cada una de las fases productivas del proyecto. 
Los resultados secuenciales obtenidos, corresponden a un enfoque territorial en tanto se logra el análisis multitemporal, multiobjetivo y multicriterio de la siguiente manera:

Visión territorial: permitió una mayor perspectiva sobre los diferentes componentes ambientales con los que cuenta el territorio a trabajar, y de esta manera ampliar la visión espacial y por ende el análisis de los recursos naturales aprovechables y óptimos para el desarrollo del proyecto, siendo esta la base del mismo.

Dinámica territorial: este análisis llevó al proyecto a idealizarse en el territorio, haciendo uso de los diferentes recursos que sobre él se encuentran, y de esta manera tener una idea más clara acerca del desarrollo y factibilidad del proyecto en la zona y viceversa, es decir, interpretar la participación y reacción del territorio en el proyecto. Es así como la industria cauchera al ser tecnificada garantiza la sostenibilidad y sustentabilidad del medio natural y social del municipio, contribuyendo al desarrollo económico del mismo.

Gestión del proyecto en el territorio: este permitió establecer el ci- clo productivo del proyecto de caucho, desde la fase inicial hasta la compensación sobre el territorio a partir de siembra de árboles en una zona específica del municipio. Gracias a los SIG se logró delimitar las zonas en las cuales es posible desarrollar cada una de las fases del ciclo reconociendo de esta manera las áreas más óptimas para la ejecución del mismo. Además, a partir de la matriz del análisis de uso y aprovechamiento de los recursos naturales se logró estimar el uso y oferta de los componentes ambientales presentes en el territorio, predominando el aprovechamiento del recurso de acuerdo a la fase del ciclo productivo. A nivel general, la inversión del proyecto se encuentra en un rango de moderada a baja debido a que en la mayoría de las dimensiones los recursos serán aprovechados y utilizados en cada uno de las diferentes etapas de las fases del ciclo productivo.

Gestión Integral del Territorio: utilizando la herramienta SIG se logró estimar las áreas potenciales del territorio en correspondencia con la visión territorial, lo que se traduce en una predominancia de áreas potenciales medias, e indica que el municipio presenta condiciones moderadamente óptimas 
para la realización del proyecto, garantizando así la factibilidad del mismo. El análisis espacial de las condiciones y elementos funcionales del municipio que resultan importantes para el proyecto, hacen parte integral de la planificación y desarrollo del proyecto al vincular y respetar las costumbres, culturas y tradiciones de la población nativa del lugar. La planeación normoestratégica le brinda al proyecto una serie de criterios de orden legal ya que establece límites y controles en el territorio frente a la normatividad. Es evidente la predominancia de la demanda legal media permitiendo al proyecto no sólo visualizar aquellas dimensiones en las que las normas son escazas, sino a su vez un análisis espacial.

La gestión integral de un proyecto productivo no solo requiere de un análisis endógeno, sino de la consideración de diversos criterios exógenos, como los grupos de interés y las variables del entorno. Este asunto corrobora la necesidad de diseñar modelos territoriales como marco para el desarrollo de proyectos productivos en zonas con alta oferta ambiental, que no sólo deben ser usados por las generaciones actuales en proyectos puntuales, sino por las generaciones venideras que podrán darle continuidad a proyectos productivos, con las compensaciones y gestión integral óptima.

La gestión integral permite un mejor manejo de los diferentes recursos naturales a emplear, ya que con el estudio y el análisis exhaustivo que requiere (apoyada actualmente de los SIG) concede de forma más consciente y real la factibilidad y sostenibilidad del proyecto. Por su parte, la evaluación multicriterio permite establecer un análisis más detallado de la zona de estudio, aplicando de manera sostenible la gestión ambiental a través de diversas etapas que al consolidarse finalmente dan lugar a la decisión más importante del proyecto. La factibilidad se estima en la viabilidad del proyecto, estableciendo una zona exacta para el desarrollo y ejecución del mismo. Los Sistemas de Información Geográfica (SIG) resultan ser una herramienta primordial para el desarrollo de cualquier proyecto, no solo por los insumos espaciales que con él se pueden generar, sino (siendo lo más valioso de esto) por el análisis espacial que permite una visión más detallada y concreta de lo que sería la zona más óptima para la ejecución del proyecto en todas sus etapas. 


\section{Referencias}

Alberti, M. and Waddell, P. (2000). An Integrated Urban Development and Ecological Simulation Model. Integrated Assessment, 1(3), 215-227.

Alcaldía de San José del Guaviare. (2011). Plan Básico de Ordenamiento territorial de San José del Guaviare. Recuperado de http://cdim.esap.edu.co/BancoMedios/Documentos\%20 PDF/pbot\%20-\%20san\%20jose\%20del\%20guaviare\%20-\%20analisis\%20territorial.pdf

Altervista. (2009). Reciclaje de llantas, tecnología México. Recuperado de http://es.scribd.com/ doc/287918744/Diagramas-de-Flujo-proceso-del-GCR\#scribd

Departamento Administrativo Nacional de Estadística, DANE. (2005). Boletín DANE, Censo General San José del Guaviare. Colombia.

ECPA, Energy and Climate Partnership of the Americas. (s.f.). Initiatives. Producción en Ciclo Cerrado en las Américas (2009-2016). Recuperado de http://www.ecpamericas.org/ Initiatives/default.aspx?id=63

Fernández, R. (2013). La dimensión económica del desarrollo sostenible. Alicante: Editorial Club Universitario.

Goodyear. (s.f.). Componentes y fabricación de un neumático. Recuperado de http://www. goodyear.com.ar/tire_know/making_a_tire/

Instituto de Hidrología, Meteorología y Estudios Ambientales de Colombia, IDEAM. (2014). Características climatológicas de ciudades principales y municipios turísticos. Recuperado de http://www.ideam.gov.co/documents/21021/21789/1Sitios+turisticos2.pdf/cd4106e9d608-4c29-91cc-16bee9151ddd\#page28

Integrate Urban Planing. (2007). International Cooperation. Recuperado de http://www. bth.se/exr/market.nsf/(WebFiles)/B0F816E525973D21C12571A000317865/\$FILE/ IntegUrbanPlan_inv-07_low.pdf

Martínez, H. (2005). La cadena del Caucho en Colombia una mirada global de su estructura y dinámica 1991-2005. Ministerio de Agricultura y Desarrollo Rural Observatorio Agrocadenas Colombia [Documento de Trabajo No. 63].

Mena, C., Gajardo, J. y Ormazábal, Y. (2006). Modelación espacial mediante geomática y evaluación multicriterio para la ordenación territorial. Rev. Fac. Ing. - Univ. Tarapacá, 14(1), 81-89.

Ministerio de Ambiente y Desarrollo Sostenible. (2013). Certificado de Incentivo Forestal CIF 2013. Recuperado de https://www.minagricultura.gov.co/tramites-servicios/apoyosincentivos/Paginas/v1/Certificado-de-Incentivo-Forestal-CIF-2013.aspx 
Ministerio del Interior. (2005). Plan de Vida Indigena, Resguardo Indígena de Barracon. Recuperado de https://www.mininterior.gov.co/sites/default/files/plan_de_vida_resguardo _indigena_barrancon_san_jose_del_guaviare_guaviare.pdf

Ministerio de Tecnologías de la Información y las Comunicaciones. (2013). El departamento del Guaviare y su capital San José del Guaviare, ahora Viven Digital. Recuperado de http:// www.mintic.gov.co/portal/604/w3-article-2001.html

Montoya, G. (2016). Propuesta del análisis integral y multicriterio de la sostenibilidad [Investigación Postdoctoral].

Posada, A., Paredes, A. y Ortiz, E. (2016). Enfoque sistémico aplicado al manejo de parques metropolitanos, una posición desde Bogotá D.C. - Colombia. Rev. U.D.C.A Act. \& Div. Cient., $19(1), 207-217$.

Posada, A. y Berrocal, A. (2015). Reconfiguracion regional en el marco del ordenamiento territorial; Francia, un espejo para Colombia. Rev. U.D.C.A Act. \& Div. Cient., 18(1), 271281.

Sandström, U., Angelstam, P. y Khakee, A. (2016). Urban comprehensive planning - identifying barriers for the maintenance of functional habitat networks. Landscape and Urban Planning, 75(1-2), 43-57. 
Recepción: 14 de junio de 2016 Evaluación: 28 de noviembre de 2016

Aprobación: 2 de diciembre de 2016 